\title{
Artículo
}




\section{LA INVESTIGACIÓN EDUCATIVA EN LA DÉCADA DEL 80: UN ESFUERZO CUALITATIVO}

Martha Cecilia Herrera $C^{*}$.

La producción investigativa en educación en los años 80 presenta un gran dinamismo y aunque su tratamiento temático y la calidad de los trabajos muestran cierto grado de heterogeneidad, este campo registra avances destacados en relación con las décadas anteriores. A dicha producción han contribuido profesionales de distintas disciplinas que marcan con el sello de su respectiva formación, la escogencia e interpretación de las dimensiones del fenómeno educativo. Sociólogos, economistas, historiadores, antropólogos, pedagogos, físicos, matemáticos, participan en los diversos equipos en los que se llevan a cabo investigaciones de carácter teórico, histórico, descriptivo, de diagnóstico o empírico, que tienden a precisar las necesidades básicas del sistema educativo.

El apoyo institucional dado a esta producción proviene de las universidades públicas y privadas, de organismos estatales y entidades de carácter privado, sin que exista ninguna coordinación entre estos esfuerzos, factor que incide en la dispersión temática, en el desperdicio de recursos y en la ausencia de retroalimentación académica e investigativa.

En relación con décadas anteriores las preocupaciones temáticas sufrieron algunas variaciones, puesto que en ciertos círculos se emprendieron estudios puntuales que se alejan de diagnósticos generales o de enfoques macrosociales, para pasar a caracterizar con mayor detenimiento los procesos educativos o sus actores desde un enfoque cualitativo. En este sentido se pueden destacar los aportes hechos desde la etnografía socioeducativa por investigadores como Rodrigo Parra, Araceli de Tezanos, Leonor Zubieta, quienes han avanzado en precisar las interacciones que se llevan a cabo en el aula. Los trabajos de los hermanos de Zubiría, abordan desde la sicogenética y la sociogenética, la conformación de las estructuras conceptuales y su incidencia en el aprendizaje de las Ciencias Sociales, al tiempo que el equipo de investigación de la Universidad Nacional, que orienta Carlos Federici, se plantea el problema de la formación de una actitud científica en Matemáticas y Ciencias Naturales. ${ }^{27}$

Desde otro ángulo continuaron saliendo a la luz los productos de la investigación sobre "Las prácticas pedagógicas en Colombia", que constituye un importante esfuerzo por

\footnotetext{
* Socióloga y magíster en Historia. Docente e investigadora de la Universidad Pedagógica Nacional.

${ }^{27}$ Rodrigo Parra. La escuela inconclusa. Plaza y Janes, Bogotá, 1986; Los maestros colombianos, Plaza y Janes, Bogotá. 1986; Pedagogía de la desesperanza. Plaza y Janes, Bogotá, 1989.

Araceli de Tezanos, Guillermo Muñoz, Emiliano Romero. Escuela y comunidad. Un problema en sentido. CIUP-UPN, Bogotá, 1982; Araceli de Tezanos. 'La escuela primaria: Una perspectiva etnográfica”. Revista Colombiana de Educación, número 8. Bogotá, 1981, pp.63-90.
}

Leonor Zubieta y Rodrigo Parra. La imagen del maestro en la escuela campesina. CI U P- LT PN, Bogotá, 1981; Leonor Zubieta. "Etnografía y política educativa". Revista Colombiana de Educación, número 10. Bogotá, 1982, pp. 107-115.

Miguel yJulián de Zubiría. Pedagogía conceptual, una propuesta curricular para la enseñanza de las Ciencias Sociales para pensar. Presencia-Canapro, Bogotá. 1986.

A. Mockus, C.A. Hernández, J. Granés, J. Charum, M.C. Castro. Las fronteras de la escuela, infbrme final proyecto ColcienciasCindec-UN, Universidad Nacional, Bogotá, 1988. 
sistematizar un área del saber, la Pedagogía; pocas son las investigaciones que se acometen de manera colectiva buscando formar escuela, equipos que emprendan análisis conjuntos con una unidad conceptual y metodológica, éste es uno de los aspectos más relevantes del proyecto que contó con los recursos financieros para emprender una obra de tal envergadura, dejando atrás el quehacer individual tan común en la tradición investigativa del país ${ }^{28}$. La persistente preocupación de este equipo por esclarecer el estatuto del maestro y la multiplicidad de sus prácticas, también inquietó a otros especialistas quienes desde distintos enfoques se interesaron por la historia y situación actual del cuerpo magisterial; aquí se pueden mencionar los trabajos de Rafael Flórez, César Vera, Francisco Parra, Guillermo Londoño, Martha Herrera ${ }^{29}$. En el conjunto de estas elaboraciones puede señalarse, además, la existencia de un interés de carácter práctico asociado a la fuerza que el movimiento magisterial y el movimiento pedagógico registró en la última década, lo cual revirtió en la preocupación por ahondar los análisis pedagógicos, dotanto al magisterio de un corpus teórico que le dé solidez como fuerza motriz en los procesos educativos y que le ayude a reconstruir su identidad como sujeto, la especificidad de su oficio, su tradición histórica y proyección social.

Por su parte las investigaciones sobre educación comunitaria y educación de adultos, encontraron impulso en entidades como el CINEP y el Instituto de Educación Permanente de la Universidad Pedagógica Nacional, en donde se destacan los proyectos de Marcos Raúl Mejía y Mario Sequeda, inscritos dentro de la metodología de la investigación-acción o investigación participativa que pretende involucrar a los sectores populares en las propuestas a desarrollar. Igualmente, Dimensión Educativa ha puesto en marcha proyectos de gran significación en el campo de la educación popular, en donde cabe mencionar los trabajos de Germán Mariño ${ }^{30}$.

El campo de la Historia de la Educación aunque sigue siendo débil también contó con progresos; a nivel general fue incluido en obras históricas de carácter global o regional como la Nueva Historia de Colombia o la Historia de Antioquia, en donde se incluyeron capítulos que dan cuenta de su dinámica; igualmente se publicaron textos como el de Aline Helg sobre la educación entre 1918 y 1957 o el de Frank Safford sobre la formación de una élite técnica $y$ empresarial en el siglo XIX, en donde se hacen sugestivas apreciaciones en este campo ${ }^{31}$. A su vez, distintos estudiosos emprendieron el análisis

\footnotetext{
${ }^{28}$ Algunos de los textos publicados por este equipo son los siguientes: Alberto Martínez Boom. El maestro y la instrucción pública en el Nuevo Reino de Granada, 1767-1809. CIUP-UPN, Bogotá, 1981; Renán Silva y Alberto Martínez. Dos estudios sobre educación en la Colonia. CIUP-UPN, Bogotá. 1984; Alberto Echeverry. Proceso de constitución de la instrucción pública, 18)91835. Universidad de Antioquia, Medellín, 1984; Olga Lucía Zuluaga. El maestro y el saber pedagógico en Colombia, 1821 -1848. Universidad de Antioquia, Medellín, 1984; Pedagogía e Historia. Foro Nacional por Colombia, Bogotá, 1987; Humberto Quiceno. Pedagogía Católica y escuela activa en Colombia, ;900-1935. Foro Nacional por Colombia, Bogotá, 1987.

${ }^{29}$ Rafael Flórez y Enrique Batista. El pensamiento pedagógico de los maestros. Copiyepes, Medellín, 1982.
}

César Vera y Francisco Parra. Contribución a un análisis de la capacitación docente y la práctica pedagógica. UPN, Bogotá. 1985; La formación de maestros polivalentes. CIUPUPN, Bogotá, 1988.

Guillermo Londoño y otros. La capacitación de los docentes en ejercicio del departamento de Antioquia e impacto de los talleres pedagógicos como estrategia de formación. Universidad de Antioquia, Medellín. 1988.

Martha Cecilia Herrera. Hacia la profesionalización del Magisterio en Colombia: 1930-1950. Bogotá.

${ }^{30}$ Mario Sequeda. La alfabetización y post-alfabetización en Colombia 1 980-1985. Balance y perpectivas. Bogotá, 1986.

Marcos Raúl Mejía. Educación popular, problemática actual. Revista Educación y Cultura, número 12. Bogotá, 1987.

Germán Mariño. Escritos sobre escritura. Dimensión Educativa, Bogotá, 1988.

${ }^{31}$ Alvaro Tirado (ed.). Nueva Historia de Colombia. Planeta, Bogotá, 1988. 8 vols.

Jorge Orlando Melo (ed.). Historia de Antioquia. Suramericana de Seguros, Bogotá, 1988.

Digitalizado por RED ACADEMICA 
histórico y sociológico en torno a instituciones de enseñanza técnica, al tiempo que se avanzó en el tratamiento de períodos específicos o en precisar la incidencia de la Iglesia Católica en el ámbito educativo ${ }^{32}$. Al igual que en otros terrenos de la producción historiográfica, estos intentos procuraron superar los enfoques tradicionales, bajo la influencia que nuevas generaciones han imprimido al modo de hacer historia, con el acopio de otras preocupaciones y paradigmas interpretativos.

La Sociología de la Educación, una de las disciplinas que ha mostrado interés en la investigación educativa desde la década del 60, continúa haciendo aportes; ya se mencionó a Rodrigo Parra cuya prolífica obra aborda problemas sobre el maestro, la escuela, la universidad; igualmente hay que destacar los trabajos de Gonzalo Cataño, así como sus esfuerzos de difusión respecto a las grandes temáticas de la sociología educativa desde la Revista Colombiana de Educación. También es preciso evocar los ensayos de Angel Facundo en torno a la calidad de la educación, de Bernardo Restrepo sobre formación de docentes y facultades de educación, y los de Julio Puig referidos a educación y lenguaje y a enseñanza técnica ${ }^{33}$. Sociólogos y economistas jugaron un papel importante en la conformación de equipos de investigación para el diagnóstico y diseño de políticas educativas en el país.

La investigación educativa encontró en este decenio canales de discusión y difusión en distintos eventos de carácter académico, como son el segundo y tercer Seminario

Aline Helg. La educación en Colombia, 1918-1957. Una historia social, económica y política. CEDEC, Bogotá, 1987.

Frank Safford. El ideal de lo práctico, el desafió de formar una élite técnica y empresarial en Colombia. Ancora-Universidad Nacional. Bogotá, 1989.

${ }^{32}$ Jane M. Loy. "Los ignorantistas y las escuelas: Oposición a la reforma educativa durante el Federalismo". Revista Colombiana de Educación, número 9 (Bogotá) 1982, pp. 9-24.

Oscar Jaimes y Jorge Eliécer Rozo. La presencia de la Escuela Nueva en Colombia: El Gimnasio Moderno. T. Posgrado de Investigación Socioeducativa, UPN, Bogotá, 1986.

Martha Cecilia Herrera y Carlos Low. La Escuela Normal Superior: 1936-1951. Proyecto de investigación en curso, UPN, Bogotá.

Alejandro Alvarez. La escuela y la instrucción pública en Bogotá, siglo XIX. I Coloquio de docentes e investigadores en Historia de la Educación, Bogotá, 2 al 5 de agosto de 1989.

Orieta López. La Escuela de Artes y Oficios de Antioquia. Inicio de la formación tecnológica. I Coloquio de docentes e investigadores en Historia de la Educación.

Catalina Reyes. Relaciones entre la cultura de la intolerancia y el control clerical de la educación. Influencia de la Iglesia sobre la educación en Colombia, 1850-1957. I Coloquio de docentes e investigadores en Historia de la Educación.

Diana Soto. La administración al servicio de la Academia. Polémica de catedráticos en la ciudad de Santa Fe, siglo XVIII. I Coloquio de docentes e investigadores en Historia de la Educación.

${ }^{33}$ Gonzalo Cataño. "Desarrollo de la investigación sociológica sobre educación en Colombia".Revista del Centro de Estudios Educativos, vol. 14, número 1. México, 1974, pp. 53-70; Educación y estructura social. Plaza y Janes, Bogotá, 1989. Ver además Revista Colombiana de Educación, 1978-1989.

Angel Facundo y Carlos Rojas. La calidad de la educación secundaria, lo que dice la investigación. M.E.N./Fondo Editorial, Bogotá, 1982; La calidad de la educación o la satisfacción de las necesidades sociales por medio de la educación. V Congreso Nacional de Sociología, Medellín, 28 al 30 de mayo de 1985.

Bernardo Restrepo. Los 30 años de la Facultad de Educación de la Universidad de Antioquia y sus 4 configuraciones curriculares. Universidad de Antioquia, Medellín, 1983; "La educación a distancia, una alternativa para la formación de docentes". Revista Educación y Cultura, número 7 (Bogotá) 1985, pp. 27-31. 
Nacional de Investigación en Educación, celebrados en 1980 y 1986 respectivamente, el último de los cuales contó con más de 200 ponencias alrededor de temas como la enseñanza y el aprendizaje, la calidad de la educación, la investigación histórica en educación y pedagogía, educación y desarrollo, y planeación educativa. En 1986 el Ministerio de Educación llevó a cabo una consulta nacional bajo el título ¿Para dónde vamos en educación?, evento de carácter masivo en el que se discutieron las diversas dimensiones de la problemática educativa. La Federación Colombiana de Educadores convocó en 1987 al Congreso Pedagógico Nacional, en donde 665 delegados escucharon y debatieron un total de 257 ponencias, las cuales versaron sobre temas como los fines y la calidad de la educación, el movimiento pedagógico, la formación de los educadores, la reforma educativa, la escuela y la comunidad, y la enseñanza de asignaturas específicas. La Universidad Santo Tomás dedicó el último Congreso Internacional de Filosofía Latinoamericana a la educación, allí se dieron cita en el año de 1988 unos 500 asistentes para conocer cerca de 100 ponencias sobre la historia de las ideas pedagógicas en América Latina, filosofía de la educación y política, modelos pedagógicos y formación de docentes, filosofía de la pedagogía liberadora, presupuestos filosóficos de la educación popular y los fundamentos de la renovación curricular en Colombia.

A mediados de 1989, la Universidad Pedagógica celebró el / Coloquio de docentes e investigadores en Historia de la Educación el cual, aunque contó con una asistencia modesta y un número de ponencias limitado, representó un espacio para el intercambio entre los intelectuales que trabajan estos temas; cuatro comisiones aglutinaron los informes presentados, política educativa, historia de las ideas pedagógicas, instituciones y fundaciones educativas, metodología de la investigación y la enseñanza de la historia de la educación. Así mismo, los congresos de Historia y sociología realizados en esta década dedicaron una comisión a la temática educativa y aunque ésta ocupa una franja todavía marginal, señala no obstante la presencia de investigadores educativos en estas áreas profesionales.

La labor de difusión en cuanto a publicaciones aún no da cuenta del caudal ¡investigativo, pocas son las revistas que presentan continuidad y en materia de libros las editoriales son esquivas al compromiso sobre los temas educativos, al considerar que no existe un público cautivo de amplitud suficiente para librar los costos de inversión. Algunas de las publicaciones periódicas que condensaron el debate en los 80 son las siguientes: Revista Colombia de Educación, órgano de la Universidad Pedagógica y su centro de investigación; Estudios Educativos, revista de la asociación de Profesionales de la Educación con sede en Medellín; Educación y Cultura, publicación de la Federación Colombiana de Educadores (Bogotá); Psicología Educativa editada por el CEIPA en Medellín; Reflexiones Pedagógicas de la Facultad de Educación de la Universidad del Valle; Educarte de la Universidad San Buenaventura con sede en Cali; Educación y Ciencia de la Universidad Pedagógica y Tecnológica de Tunja; Preescolar editada en la Facultad de Educación de la Universidad Industrial de Santander; Educación Hoy, revista de la Confederación Interamericana de Educación Católica y la Universidad Javeriana. La Facultad de Educación de la Universidad de Antioquia acaba de lanzar la Revista Educación y Pedagogía, para reanudar una edición que tuvo su origen en el año de 1954 en dicha facultad. Es preciso señalar que estas publicaciones tienen grandes deficiencias en su circulación, lo que hace que su radio de acción sea reducido y de carácter local. La Universidad de Antioquia, la Universidad Santo Tomás, la Universidad Pedagógica, la fundación Foro por Colombia, han dado apoyo institucional a la edición de libros sobre educación y en el último año la Cooperativa Nacional del Magisterio publicó algunos textos educativos. De otro lado, el Boletín Cultural y Bibliográfico del Banco de la República ha dedicado algunas de sus páginas a la reseña de libros sobre educación. 
Pese a las limitaciones, es un hecho que el tema educativo ha gozado del boom editorial registrado en el país en la última década.

La Red Latinoamericana de Información y Documentación en Educación, REDUC, reúne, resume y microficha los documentos que sobre el tema se producen en los respectivos países, permitiendo la sistematización y consulta bibliográfica a estudiantes e investigadores, así como el intercambio de información en América Latina; en Colombia el REDUC ha publicado ocho volúmenes en los cuales se compilaron cerca de 700 documentos sobre educación en el país. A su vez, la puesta en marcha por parte del ICFES del Sistema Nacional de información y Documentación para la Educación Superior, SIDES, jugará un papel importante en lo que respecta a la red informativa en educación.

Además de los logros que presenta el panorama anteriormente descrito, la investigación educativa tiene ante sí ausencias y retos que deberá llenar y enfrentar, para lograr desarrollos significativos en las próximas décadas. Por un lado, el agotamiento de los paradigmas interpretativos que primaron en los decenios del 60 y 70 -teoría del capital humano y reproductivista-, que pusieron el énfasis en los resultados finales de la educación y en los enfoques macrosociales, aún no logra ser reemplazado por un paradigma consistente, pese a los avances hechos en el 80 por privilegiar el análisis de los procesos y de los actores sociales involucrados en la educación. En este sentido es necesario enfatizar la importancia que tiene la generalización del debate y la polémica entre los distintos núcleos comprometidos en la investigación, que deberá estar exenta de actitudes de autosuficiencia e intolerancia intelectual. Lo anterior también se corresponde con la inminencia de unificar el acervo investigativo, que permita la acumulación de dicho conocimiento y supere la atomización de los esfuerzos. De igual modo otro reto que presenta la investigación educativa, es el lograr incidir en los procesos actuales de la educación nacional, tanto en lo que se refiere a la planeación y ejecución de políticas educativas, como al cuestionamiento y transformación de las prácticas pedagógicas de los sectores que interactúan en este espacio social. En síntesis, logros y ausencias, avances y obstáculos, arrojan un balance satisfactorio sobre el quehacer investigativo en educación que permite hablar de aciertos de carácter cualitativo en el transcurso de la presente década. 